\title{
SOFT block (Sciatic, Obturator and $F$ emoral nerve block Technique): a single-puncture, sole anesthetic for knee surgery
}

\author{
Ahmad Muhammad Taha, MD • Nader Darwich, MD
}

Received: 9 June 2017/Revised: 25 July 2017/Accepted: 10 August 2017/Published online: 18 August 2017

(c) Canadian Anesthesiologists' Society 2017

\section{To the Editor,}

Peripheral nerve block can provide safe, effective anesthesia for knee surgery. In some cases, however, it is necessary to block the femoral nerve, sciatic nerve, and obturator nerves. ${ }^{1}$ Multiple individual nerve blocks are time consuming and may cause patient discomfort. ${ }^{2,3}$ To minimize both time and discomfort, we suggest using the $\underline{S}$ ciatic, $\underline{O}$ bturator, and $\underline{F}$ emoral nerve block $\underline{T}$ echnique i.e., the SOFT block. It is a single-puncture, supine, ultrasonography (US)-guided technique for anesthesia of the sciatic, obturator, and femoral nerves.

This prospective observational cohort study was approved by our institutional ethics board (REC 001) and was registered with ClinicalTrials.gov (NCT02951520). After informed written consent, 50 patients with torn knee ligaments and a body mass index of $<30 \mathrm{~kg} \cdot \mathrm{m}^{-2}$ underwent a SOFT block. Each participant was given 2

This work was done at: Healthpoint Hospital, Abu Dhabi, UAE. This work has not been presented at any meetings and has not been published elsewhere.

Trial Registration: www.clinicaltrials.gov, NCT02951520. Registered 29 October 2016.

A. M. Taha, MD

Department of Anesthesia, Healthpoint Hospital, Abu Dhabi, UAE

A. M. Taha, MD ( $\square)$

Department of Anesthesia, Ain Shams University, Cairo, Egypt

e-mail: ahmadtaha_1@yahoo.com

N. Darwich, MD

Department of Orthopaedics, Healthpoint Hospital, Abu Dhabi, UAE mg midazolam $i v$ and placed in supine position (with the hip abducted and externally rotated). Under aseptic conditions, a linear US probe was placed on the inguinal crease to identify the femoral nerve and vessels. Transducer orientation, needle paths, and anatomy are shown in the Figure. Using an in-plane technique, a $12-\mathrm{cm}$ stimulating block needle was inserted just medial to the femoral vein and advanced 1-3 cm just below and parallel to the skin. It was then redirected toward the lateral fibres of the femoral nerve, where $15 \mathrm{~mL}$ of local anesthetic was injected. To block the obturator nerve, the probe was moved medially, placed superior to the needle, and tilted cranially to identify the pectineus muscle proximally. The needle was subsequently withdrawn to the subcutaneous tissue and redirected using an out-of-plane technique toward the deep surface of the pectineus, where $10 \mathrm{~mL}$ of local anesthetic was injected. ${ }^{4}$ To identify the sciatic nerve, we switched to the curvilinear probe, placed it inferior to the needle, and rotated/tilted the probe to achieve the best longitudinal image of the sciatic nerve. The needle was again withdrawn subcutaneously and redirected using an in-plane technique toward the sciatic nerve deep to the inferior border of the quadratus femoris muscle. The needle tip was adjusted to elicit tibial twitches using a current of $0.5-1.0 \mathrm{~mA}$ before $20 \mathrm{~mL}$ of local anesthetic was injected. If needed, the skin at the exit of the femoral tunnel guide wire was also infiltrated with local anesthetic. No intraoperative opioids were used, although propofol $\left(1-2 \mathrm{mg} \cdot \mathrm{kg}^{-1} \mathrm{hr}^{-1}\right)$ was infused if sedation was requested. The SOFT block success rate (defined as painless surgery), performance time (including scanning and needling times), and patient discomfort (on a numerical rating scale) were recorded.

Among the 50 patients, 48 (96\%) were male. The overall median [interquartile range (IQR)] age was 27 [22-35] yr, 

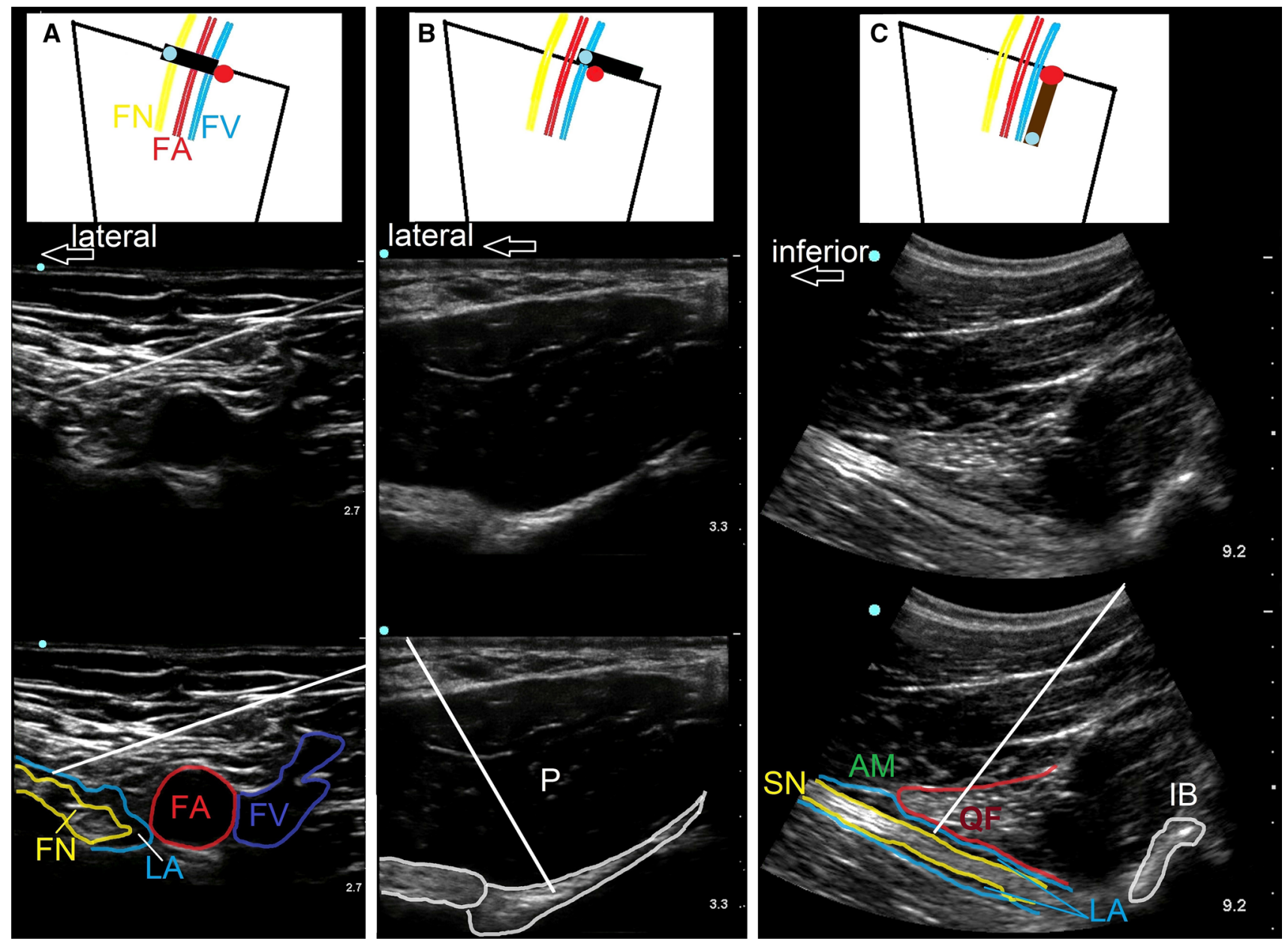

Figure SOFT block technique. A) The linear probe (black rectangle) is placed on the inguinal crease to identify the femoral nerve (FN). The needle is inserted (red dot) just medial to the femoral vein (FV) and advanced laterally toward the lateral FN fibres. B) To block the obturator nerve, the probe is moved medially (superior to the needle) and tilted cranially. Using an out-of-plane technique, the needle is redirected toward the interfascial plane deep to the pectineus $(\mathrm{P})$ muscle. C) Finally, the curved probe (brown rectangle) is placed vertically, inferior to the needle, to identify the sciatic nerve (SN), which was blocked deep to the quadratus femoris (QF) muscle, which appeared as the "lesser than" sign $(<)$, with the apex directed inferiorly. $\mathrm{AM}=$ adductor magnus; FA, FN, and FV = femoral artery, nerve, and vein respectively; $\mathrm{IB}=$ ischium bone; $\mathrm{LA}=$ local anesthetic; $\mathrm{P}=$ pectineus; $\mathrm{QF}=$ quadratus femoris; blue dots $=$ probe orientation markers; white lines $=$ simulated needle paths. SOFT $=$ Sciatic, Obturator and Femoral nerve block Technique and the median [IQR] body mass index was 26.4 [23.7-29.7] $\mathrm{kg} \cdot \mathrm{m}^{-2}$. Altogether, 45 (90\%) participants had successful SOFT blocks that provided complete anesthesia. The five (10\%) participants with incomplete blocks reported incomplete motor block of the sciatic nerve, but complete blocks of the obturator and femoral nerve distributions. The median [IQR] participant discomfort on a numeric rating scale of $1-10$ was 2 [0-3], and the median [IQR] performance time was 5.5 [4.7-6.6] $\mathrm{min}$.

The success rate of the SOFT block was comparable to that of a previously described approach for blocking the sciatic, femoral, and obturator nerves while taking less time than the 25 min reported. ${ }^{3}$ Although the sciatic nerve is a deep structure, longitudinal scanning facilitates its identification. ${ }^{5}$ To obtain an uninterrupted longitudinal image of the sciatic nerve, the probe was placed just medial to the femoral vessels. This site permitted easy access to the obturator nerve as well as proximal and distal sciatic nerve segments. Even though the sciatic nerve is easier to identify distally, we chose to block it proximally, deep to the quadratus femoris, to anesthetize the semitendinosus muscle. During the block of the femoral nerve, we took care to avoid the femoral vessels, thereby reducing the risk of vascular injury. We do not recommend the SOFT block for inexperienced operators.

Our experience suggests that, in appropriately selected patients and operators, the simple positioning, minimal patient discomfort, and short performance time found with 
the SOFT block may make it an effective anesthetic technique for knee surgery.

Acknowledgements We sincerely thank Professor Dr A. El Makhzangy (Professor of ENT, Ain Shams University), Dr. M. Mohanan (anesthesia specialist, Healthpoint Hospital), Miss L. Ahmad (RN, Healthpoint Hospital), and the staff of Healthpoint Hospital for their tremendous help.

Conflicts of interest No conflict of interest exists. Study equipment support was provided by departmental sources.

Editorial responsibility This submission was handled by Dr. Gregory L. Bryson, Deputy Editor-in-Chief, Canadian Journal of Anesthesia.

Funding None. Study equipment support was provided by departmental sources.

\section{References}

1. Taha AM, Abd-Elmaksoud AM. Arthroscopic medial meniscus trimming or repair under nerve blocks: Which nerves should be blocked? Saudi J Anaesth 2016; 10: 283-7.

2. Fanelli G, Casati A, Garancini P, Torri G. Nerve stimulator and multiple injection technique for upper and lower limb blockade: failure rate, patient acceptance, and neurologic complications. Study Group on Regional Anesthesia. Anesth Analg 1999; 88: 847-52.

3. Ota J, Sakura S, Hara K, Saito Y. Ultrasound-guided anterior approach to sciatic nerve block: a comparison with the posterior approach. Anesth Analg 2009; 108: 660-5.

4. Taha AM. Ultrasound-guided obturator nerve block: a proximal interfascial technique. Anesth Analg 2012; 114: 236-9.

5. Tsui BC, Ozelsel TJ. Ultrasound-guided anterior sciatic nerve block using a longitudinal approach: "expanding the view". Reg Anesth Pain Med 2008; 33: 275-6. 